Review

\title{
Latest Overview of the Cyclin-Dependent Kinases 4/6 Inhibitors in Breast Cancer: The Past, the Present and the Future
}

\author{
Xiu Chen ${ }^{1 *}$, Di Xu1 ${ }^{*}$, Xingjiang $\mathrm{Li}^{2 *}$, Jian Zhang${ }^{1}$, Weilin $\mathrm{Xu}^{1}$, Junchen Hou${ }^{1}$, Wei Zhang ${ }^{1}$, Jinhai Tang ${ }^{1 凶}$ \\ 1. Department of General Surgery, the First Affiliated Hospital of Nanjing Medical University, Nanjing, Jiangsu, China. \\ 2. Changzhou Wujin People's Hospital, Changzhou, Jiangsu, China. \\ * Contributing equally to this article
}

$\triangle$ Corresponding author: Jinhai Tang, Department of General Surgery, the First Affiliated Hospital of Nanjing Medical University, Guangzhou Road 300, Nanjing, Jiangsu, 210029, China. Email: jhtang@njmu.edu.cn

(c) The author(s). This is an open access article distributed under the terms of the Creative Commons Attribution License (https://creativecommons.org/licenses/by/4.0/). See http://ivyspring.com/terms for full terms and conditions.

Received: 2019.01.13; Accepted: 2019.08.26; Published: 2019.10.21

\begin{abstract}
Endocrine resistance in hormone receptor positive breast cancer patients urges us to develop novel approaches such as inhibitors of the cyclin-dependent kinases (CDK) 4/6 to reverse its resistance. Nowadays, three selective CDK4/6 inhibitors (Palbociclib, Ribociclib and Abemaciclib) are approved by Federal Drug Administration and the European Medicines Agency for the treatment of advanced and metastatic HR+/HER2- breast cancer. However, no consistent conclusion has been reached to its application in other types of breast cancer. Therefore, the purpose of our study was to overview the clinical trials about the beneficial effects of Palbociclib, Ribociclib and Abemaciclib in breast cancer with their tolerable adverse effects, and discuss their resistant mechanisms thus looking for useful biomarkers to predict the efficiency of the CDK4/6 inhibitors. The CDK4/6 inhibitors application after the support of preclinic and clinic data will be helpful to provide other alternatively suitable strategies for different types of breast cancer patients.
\end{abstract}

Key words: breast cancer, drug, cyclin-dependent kinases 4/ 6 inhibitors, adjuvant therapy, neoadjuvant therapy

\section{Introduction}

For hormone receptor positive $(\mathrm{HR}+)$ breast cancer patients, primary or secondary endocrine therapy resistance is an urgent challenge in recent time. Therefore, approaches targeting other pathway small molecules may prevent and delay the endocrine resistance. Indeed, the combination of the steroidal aromatase-inhibitor (Exemestane) and the mTORinhibitor (Everolimus) enhances efficacy of endocrine therapy although with increased toxicity. At the same time, inhibitors of the cyclin-dependent kinases (CDK) 4/6 will delay and reverse endocrine resistance with tolerable adverse effects $[1,2]$.

It is well acknowledged that CDK4/ 6 inhibitors mainly block retinoblastoma tumor suppressor protein phosphorylation, thus preventing progression through the cell cycle.
For several decades, three drugs of the selective CDK4/6 inhibitors have been approved by the Federal Drug Administration (FDA) and the European Medicines Agency (EMA) for the treatment of advanced and metastatic HR+/HER2- breast cancer. For instance, Palbociclib (PD0332991)[3] provides no activities against 36 additional kinases and Ribociclib (LEE011) is in lack of activities against CDK1, 2. These two agents are approved for first line treatment in combination with Letrozole. While Palbociclib and Abemaciclib (LY2835219) with attenuated potency against CDK1, 2, 7, 9 are registered for second line therapy with Fulvestrant [4-8].

In spite of numerous completed studies considering the pharmacokinetic activity, safety and 
efficacy of these three selective CDK4/ 6 inhibitors, a plenty of ongoing clinical trials are investigating the indispensable position of Palbociclib, Ribociclib and Abemaciclib in different subtypes of breast cancer.

In this review, we mainly overviewed the clinical trials about the beneficial effects of Palbociclib (Table 1), Ribociclib (Table 2) and Abemaciclib (Table 3) in breast cancer with their tolerable adverse effects, and discussed their resistant mechanisms thus looking for useful biomarkers to predict the efficiency of the CDK4/6 inhibitors. Preclinic and clinic data will be helpful to provide alternatively suitable strategies for different types of breast cancer patients.

\section{Application of CDK4/6 inhibitors in HR+/HER2- advanced breast cancer}

Palbociclib, Abemaciclib and Ribociclib all generated significant improvements in median progression free survival (PFS) when combined with an endocrine drug for the hormone receptor-positive advanced breast cancer patients $[9,10]$.

The Phase III MONALEESA-2 trial (NCT01958021) enrolled 668 postmenopausal HR+/HER2- sub-type breast cancer patients, among them 295 patients were over 65 years old. Randomly, they were randomly grouped to take Ribociclib (600mg/day, 3-weeks-on/1-week-off) as well as Letrozole $(2.5 \mathrm{mg} /$ day $)$ as the first-line therapy or placebo and Letrozole until the endpoints. Data summarized that Ribociclib plus Letrozole obviously improved PFS of breast cancer patients, with no different effects between older and younger patients $[11,12]$. Furthermore, in the US, a three-year data of 263 members showed that Ribociclib plus Letrozole seemed to be an effective and economical approach for HR+/ HER2- advanced breast cancer patients[13, 14].

Table 1. Studies of Palbociclib

\begin{tabular}{|c|c|c|c|c|c|c|c|}
\hline Study name/ID & Phase & Subtype & Prior treatment & Allocation & Number & Combination drugs & Aims \\
\hline $\begin{array}{l}\text { PATHWAY/ } \\
\text { NCT03423199 }\end{array}$ & 3 & $\mathrm{HR}+/ \mathrm{HER} 2-\mathrm{ABC}$ & N/A & Non-Randomized & 180 & Tamoxifen \pm Goserelin & PFS,OS \\
\hline $\begin{array}{l}\text { PASIPHAE/ } \\
\text { NCT03322215 }\end{array}$ & 2 & $\mathrm{HR}+/ \mathrm{HER} 2-\mathrm{ABC}$ & endocrine & Randomized & 196 & Fulvestrant & PFS,OS \\
\hline $\begin{array}{l}\text { INGE-B/ } \\
\text { NCT02894398 }\end{array}$ & 2 & $\mathrm{HR}+/ \mathrm{HER} 2-\mathrm{ABC}$ & $\mathrm{N} / \mathrm{A}$ & Non-Randomized & 360 & none & CBR \\
\hline NCT02668666 & 2 & $\mathrm{HR}+/ \mathrm{HER} 2-\mathrm{ABC}$ & $\mathrm{N} / \mathrm{A}$ & Non-Randomized & 71 & Tamoxifen & Response Rates,PFS,CBR,OS \\
\hline $\begin{array}{l}\text { PRECYCLE/ } \\
\text { NCT03220178 }\end{array}$ & 4 & $\mathrm{HR}+/ \mathrm{HER} 2-\mathrm{ABC}$ & $\mathrm{N} / \mathrm{A}$ & Non-Randomized & 960 & none & DQoL,PFS, OS \\
\hline NCT03425838 & 3 & $\mathrm{HR}+/ \mathrm{HER} 2-\mathrm{ABC}$ & $\mathrm{N} / \mathrm{A}$ & Non-Randomized & 1050 & AI or Fulvestrant & PFS2,OS \\
\hline $\begin{array}{l}\text { PYTHIA/ } \\
\text { NCT02536742 }\end{array}$ & 2 & $\mathrm{HR}+/ \mathrm{HER} 2-\mathrm{MBC}$ & endocrine & Non-Randomized & 120 & Fulvestrant & PFS \\
\hline NCT02384239 & 2 & $\mathrm{HR}+/ \mathrm{HER} 2-\mathrm{MBC}$ & N/A & Non-Randomized & 70 & none & Tumor Progression,PFS \\
\hline $\begin{array}{l}\text { PACE/ } \\
\text { NCT03147287 }\end{array}$ & 2 & $\mathrm{HR}+/$ HER2- MBC & endocrine & Randomized & 220 & $\begin{array}{l}\text { Fulvestrant and Avelumab } \\
\text { or Fulvestrant }\end{array}$ & PFS,OSR \\
\hline $\begin{array}{l}\text { PATRICIA/ } \\
\text { NCT02448420 }\end{array}$ & 2 & $\mathrm{HR}+/ \mathrm{HER} 2+\mathrm{MBC}$ & N/A & Non-Randomized & 138 & None & PFS \\
\hline $\begin{array}{l}\text { PATINA/ } \\
\text { NCT02947685 }\end{array}$ & 3 & $\mathrm{HR}+/ \mathrm{HER} 2+\mathrm{MBC}$ & N/A & Non-Randomized & 496 & None & PFS,OS \\
\hline $\begin{array}{l}\text { PALLAS/ } \\
\text { NCT02513394 }\end{array}$ & 3 & $\mathrm{HR}+/ \mathrm{HER} 2-\mathrm{EBC}$ & $\begin{array}{l}\text { neo/adjuvant } \\
\text { therapy }\end{array}$ & Randomized & 5600 & $\begin{array}{l}\text { standard adjuvant } \\
\text { endocrine therapy }\end{array}$ & iDFS,DRFS,LRRFS,OS \\
\hline NCT02605486 & 1,2 & $\mathrm{AR}+\mathrm{MBC}$ & $\begin{array}{l}\text { endocrine or } \\
\text { chemotherapy }\end{array}$ & Non-Randomized & 51 & Bicalutamide & PFS,Toxicity assessment \\
\hline $\begin{array}{l}\text { BRE15-024/ } \\
\text { NCT03090165 }\end{array}$ & 1,2 & $\mathrm{AR}+\mathrm{TNBC}$ & N/A & Non-Randomized & 58 & Bicalutamide & CBR,ORR \\
\hline NCT02600923 & 3 & $\mathrm{HR}+/ \mathrm{HER} 2-\mathrm{ABC}$ & none & Non-Randomized & 130 & Letrozole & $\mathrm{AE}$ \\
\hline NCT02679755 & 4 & $\mathrm{HR}+/ \mathrm{HER} 2-\mathrm{ABC}$ & none & Non-Randomized & 300 & Letrozole & $\mathrm{AE}$ \\
\hline NCT02626507 & 1 & ER+/HER2- BC & none & Non-Randomized & 18 & None & $\mathrm{AE}$ \\
\hline $\begin{array}{l}\text { PALINA/ } \\
\text { NCT02692755 }\end{array}$ & 2,3 & $\mathrm{HR}+/ \mathrm{HER} 2-\mathrm{BC}$ & N/A & Non-Randomized & 35 & Letrozole or Fulvestrant & $\mathrm{AE}$ \\
\hline NCT03401359 & $\mathrm{N} / \mathrm{A}$ & $\mathrm{HR}+\mathrm{MBC}$ & N/A & Non-Randomized & 100 & Endocrine & $\begin{array}{l}\text { Biomarker of acquired } \\
\text { resistance }\end{array}$ \\
\hline NCT03238196 & $1 b$ & $\begin{array}{l}\text { ER+/HER2-/FGFR- } \\
\text { amplified MBC }\end{array}$ & N/A & Non-Randomized & 32 & Fulvestrant and Erdafitinib & $\begin{array}{l}\text { AE,DLT,MTD,Next } \\
\text { Generation Sequencing }\end{array}$ \\
\hline NCT02774681 & 2 & HR-/HER2+ MBC & N/A & Single group & 12 & Trastuzumab & $\begin{array}{l}\text { Radiographic response rate in } \\
\text { the CNS,AE,OS,PFS,ORR }\end{array}$ \\
\hline NCT03304080 & $1 / 2$ & $\mathrm{HR}+/ \mathrm{HER} 2+\mathrm{MBC}$ & $\mathrm{N} / \mathrm{A}$ & Single group & 36 & $\begin{array}{l}\text { Anastrozole, Trastuzumab, } \\
\text { Pertuzumab }\end{array}$ & DLT,MTD,CBR,PFS,AE \\
\hline NCT03065387 & 1 & $\begin{array}{l}\text { HER2 Mutation or } \\
\text { Amplification }\end{array}$ & N/A & Non-Randomized & 120 & Neratinib & MTD \\
\hline NCT03054363 & $1 b / 2$ & $\mathrm{HR}+/ \mathrm{HER} 2+\mathrm{MBC}$ & & Single group & 25 & Tucatinib, Letrozole & AE,PFS \\
\hline $\begin{array}{l}\text { PALTAN/ } \\
\text { NCT02907918 }\end{array}$ & 2 & $\begin{array}{l}\text { Stage II-III } \\
\text { ER+/HER2+ BC }\end{array}$ & none & Single group & 48 & $\begin{array}{l}\text { Letrozole, Trastuzumab, or } \\
\text { Goserelin }\end{array}$ & Response Rates, AE, Outcomes \\
\hline
\end{tabular}


Table 2. Studies of Ribociclib

\begin{tabular}{|c|c|c|c|c|c|c|c|}
\hline Study name/ID & Phase & Subtype & Prior treatment & Allocation & Number & Combination drugs & Aims \\
\hline NCT03671330 & 2 & $\mathrm{HR}+/ \mathrm{HER} 2-\mathrm{ABC}$ & N/A & Randomized & 315 & none & PFS \\
\hline $\begin{array}{l}\text { MONALEESA-2/ } \\
\text { NCT01958021 }\end{array}$ & 3 & $\mathrm{HR}+/ \mathrm{HER} 2-\mathrm{ABC}$ & none & Randomized & 670 & Letrozole & PFS,OS,ORR,CBR \\
\hline NCT02712723 & 2 & $\mathrm{HR}+/ \mathrm{HER} 2-\mathrm{ABC}$ & $\mathrm{N} / \mathrm{A}$ & Randomized & 120 & Letrozole & PEPI rate, $\mathrm{pCR}$ \\
\hline NCT03096847 & 3 & $\mathrm{HR}+/ \mathrm{HER} 2-\mathrm{MBC}$ & N/A & Non-Randomized & 500 & None & CBR,PFS \\
\hline $\begin{array}{l}\text { AMICA/ } \\
\text { NCT03555877 }\end{array}$ & 2 & $\mathrm{HR}+/ \mathrm{HER} 2-\mathrm{MBC}$ & $\mathrm{N} / \mathrm{A}$ & Non-Randomized & 150 & None & PFS,OS,CBR \\
\hline $\begin{array}{l}\text { RIBBIT/ } \\
\text { NCT03462251 }\end{array}$ & 3 & $\mathrm{HR}+/ \mathrm{HER} 2-\mathrm{MBC}$ & N/A & Non-Randomized & 160 & None & PFS,OS,CBR \\
\hline $\begin{array}{l}\text { LEADER/ } \\
\text { NCT03285412 }\end{array}$ & 2 & $\mathrm{ER}+\mathrm{EBC}$ & N/A & Non-Randomized & 120 & Endocrine & AE, DFS \\
\hline NCT02599363 & 1 & $\mathrm{Rb}+\mathrm{ABC}$ & N/A & Non-Randomized & 28 & None & $\mathrm{AE}$ \\
\hline $\begin{array}{l}\text { MAINTAIN/ } \\
\text { NCT02632045 }\end{array}$ & 2 & HR+ HER2- ABC & N/A & Randomized & 132 & Fulvestrant & ORR \\
\hline $\begin{array}{l}\text { TRINITI-1 trial/ } \\
\text { NCT01857193 }\end{array}$ & 1 & ER+/HER2- ABC & $\mathrm{N} / \mathrm{A}$ & Randomized & 132 & $\begin{array}{l}\text { Everolimus and Exemestane or } \\
\text { Exemestane }\end{array}$ & DLT,safety and tolerability \\
\hline NCT02657343 & $1 b / 2$ & $\mathrm{HER} 2+\mathrm{ABC} / \mathrm{MBC}$ & $\mathrm{N} / \mathrm{A}$ & Non-Randomized & 86 & T-DM1 or Trastuzumab or Fulvestrant & MTD,ORR,PFS,OS \\
\hline
\end{tabular}

Table 3. Studies of Abemaciclib

\begin{tabular}{lllllll}
\hline Study name/ID & Phase & Subtype & Prior treatment & Allocation & Number & Combination drugs \\
\hline NCT03130439 & 2 & Rb+ TNMBC & N/A & Non-Randomized & 37 & N/A \\
monarchE/NCT03155997 & 3 & HR+/HER2-MBC & N/A & Randomized & 4580 & standard adjuvant endocrine therapy \\
NCT02057133 & 1 & BC & N/A & Non-Randomized & 198 & N/A \\
NCT02779751 & 1 & BC & N/A & Non-Randomized & 100 & N/A \\
monarcHER/NCT02675231 & 2 & HR+/HER2+ MBC & & Randomized & 225 & Trastuzumab, Fulvestrant
\end{tabular}

PFS: Progression Free Survival; IDFS: Invasive Disease Free Survival; OS: Overall Survival; ORR: Objective Response Rate

In the grade II/III HR+/HER2- invasive breast cancer patients, the expression of the Ki67 was dramatically decreased $(\geq 92 \%)$ in those receiving combination of Ribociclib and Letrozole comparing to those who receiving Letrozole alone (69\%)[15]. An NCT02712723 trial investigated the neoadjuvant use of Ribociclib plus Letrozole. Also, in pretreated HR+/HER2- advanced breast cancer (ABC) patients, after combination of Ribociclib plus Fulvestrant therapy, partial responses (PRs) were observed in patients with prior treatment of Fulvestrant. An ongoing NCT03671330 trial is enrolling 315 Chinese populations to compare the PFS of HR+/ HER2- ABC patients receiving Ribociclib treatment with those who receive placebo.

Fifty early-stage breast cancer patients were enrolled in a phase II neoadjuvant trial. From the results, the complete cell cycle arrest (CCCA: central Ki67 $\leq 2.7 \%$ ) rate was significantly higher in combination of Palbociclib with Anastrozole, compared to Anastrozole monotherapy [16]. Recently, the clinical trials PATHWAY, PASIPHAE, INGE-B, NCT02668666, PRECYCLE, NCT03425838 are ongoing to explore the PFS, overall survival (OS), clinical benefit rate (CBR) and quality of life (QoL) in the breast cancer patients.

A cohort of $47 \mathrm{ABC}$ patients with or without PIK3CA mutations who received Abemaciclib therapy, reached a higher disease control rate $(\mathrm{CR}+\mathrm{PR}+\mathrm{SD})$ in $\mathrm{HR}+$ patients (29 of 36 patients, $81 \%$ ) than the HR- patients (3 of 9 patients, 33\%). Meanwhile, among the HR+ group, the radiographic responses occurred exclusively, the clinical benefit rate $(\mathrm{CR}+\mathrm{PR}+\mathrm{SD} \geq 24$ weeks) was $61 \%$, median duration of response was 13.4 months, and median PFS was 8.8 months [17].

A phase I study consisting of $225 \mathrm{ABC}$ or metastatic breast cancer (MBC) patients and a phase II study including 132 patients [18] demonstrated that single-use as well as united-use of Abemaciclib were well effective in HR-positive breast cancer. More intensive studies reported an acceptably safe dose at $200 \mathrm{mg}$ Q12H of Abemaciclib when administered as a single-use in Japanese cancer patients [19].

From the results of several studies, it is easy to conclude the idea that Palbociclib, Abemaciclib and Ribociclib play important roles in the enhancement of survival periods of HR+/HER2- ABC patients. Further trials are ongoing to gather more information and better appropriate dose or combination of CDK4/6 inhibitors in this type of breast cancer.

\section{Application of CDK4/6 inhibitors in HR+/HER2- metastatic breast cancer}

Impressive activities of CDK4/6 inhibitors are demonstrated in ER+/HER2- MBC patients with a great efficacy and low toxicity [20]. Simultaneously, MBC patients with a discontinuation of CDK $4 / 6$ inhibitors are potential to undergo rapid disease progression [21]. 
Palbociclib shows great promise in the strategy of HR+ metastatic breast cancer [24]. A study of 525 post-menopausal women with HR+/HER2- MBC demonstrated that those who transferred from CDK4/6 inhibitor-based treatment to chemotherapy had more tendency to suffer from recurrent rapidly progressing disease [22].

Vered et al. carried out a Palbociclib Expanded Access Program (EAP) and collected a total of 334 metastatic ER+/HER2- breast cancer patients to prove the good tolerance and the safety of Palbociclib with Letrozole [23].

On the contrary, in face of the twenty-three HR+/HER2- MBC patients with previous use of Everolimus, the adding of Palbociclib-based therapy revealed limited clinical benefits in the PFS, objective response rate (ORR) and CBR [24]. Meanwhile, no biomarkers have been detected to define which subtype of patients will benefit from the addition of CDK4/6 inhibitors to endocrine therapy in MBC. In another words, we should not regard the ESR1 mutational status as a biomarker to restrict the use of CDK 4/6 inhibitors [25].

Therefore, more ongoing clinical trials just like PYTHIA, NCT02384239, PACE are conducted to further detect the effectiveness of Palbociclib-based therapy in $\mathrm{HR}+$ metastatic breast cancer.

Ribociclib is reported to express functional $\mathrm{Rb}$ protein for its antiproliferative activity in HR+/HER2- metastasis breast cancer [26]. Also, NCT03096847, AMICA and RIBBIT trials are discovering the PFS, OS, CBR of Ribociclib in combination with anti-hormonal Therapies.

Besides this, studies about the Abemaciclib used in metastatic breast cancer are numbered the phase II NCT03130439 and the phase III MonarchE clinical trials.

So CDK4/6 inhibitors are potentially effective therapies to promote prognoses of $\mathrm{HR}+/ \mathrm{HER} 2-$ metastasis breast cancer patients with further confirmations needed.

\section{Other types of breast cancer}

Activities of CDK4/6 inhibitors in non-luminal breast cancer types were also discussed [27-29].

For HER2+ breast cancer patients, they also gained benefits from the use of Palbociclib, Ribociclib and Abemaciclib. In a reported phase II study, two $(5 \%)$ of the 37 enrolled patients had HR+/HER2+ breast cancer, without prior HER2-directed treatment. Owing to the single application of Palbociclib, one of them had a partial response, the other had a stable disease lasting for 5 months [3].

Meanwhile, the PATRICIA (NCT02448420) study aims to evaluating the role of Palbociclib when added to the standard Trastuzumab therapy in HER2+ MBC.

Another ongoing phase 2 study (NCT02774681) is evaluating effects of Palbociclib in HR-/HER2+ breast cancer patients with brain metastases. Patients who had received more than two prior chemotherapy regimens, or had uncontrolled neurologic symptoms or leptomeningeal disease will be excluded.

NCT03304080 is discovering whether Palbociclib combined with Anastrozole, Trastuzumab, and Pertuzumab could be a first-line therapy in $\mathrm{HR}+/ \mathrm{HER} 2+\mathrm{MBC}$ patients. After the standard four to eight cycles of chemotherapy and anti-HER2 therapy, Palbociclib will be added to the following endocrine therapy in PATINA (NCT02947685) study. The results will give an answer to the investigators if the addition of Palbociclib delays disease progression of $\mathrm{HR}+/ \mathrm{HER} 2+\mathrm{MBC}$ patients. A phase 2 study (NCT03054363) is exploring the efficacy of a triple targeted drug combination (Palbociclib, tucatinib and Letrozole) in HR+/HER2+ MBC patients.

A non-randomized, phase I/II study (NCT02657343) will test maximum tolerated dose (MTD), CBR, ORR, PFS, OS and AEs of Ribociclib in combination with Trastuzumab or T-DM1 for advanced or metastatic HER2+ breast cancer patients.

In the phase I study of single Abemaciclib use, eleven HR+/HER2+ MBC patients obtained positive clinical responses. To be more precise, four $(36 \%)$ patients reached partial response and 7 (64\%) patients got stable disease [17]. The monarcHER phase II, randomized trial (NCT02675231) will soon come to a conclusion on the PFS, OS, ORR, DoR, CBR and QoL of $\mathrm{HR}+/ \mathrm{HER} 2+\mathrm{MBC}$ patients when receiving Abemaciclib and Trastuzumab as well as Fulvestrant.

A Randomized Phase III Trial (PALLAS) is recruiting 5600 participants to compare the invasive disease free survival (iDFS), distant recurrence-free survival (DRFS), locoregional recurrences-free survival (LRRFS) and OS of the collaborative use of Palbociclib with or without other therapies in early breast cancer patients. For the early stage setting of HR+/HER2+ breast cancer patients, the NA-PHER2 (a phase II, neoadjuvant study) evaluated a combination of Palbociclib, Fulvestrant, Trastuzumab and Pertuzumab in order to avoid the use of chemotherapy[30]. In this study, results of 36 patients concluded that the combination therapy had a significant effect on the expression of Ki67 at baseline ( $\mathrm{p}<0.0001$ ). Besides, $27 \%$ of patients achieved a pathologic complete response ( $\mathrm{pCR}$ ) before surgery. At the same time, PALTAN (NCT02907918) trial is evaluating the $\mathrm{pCR}$ rate of newly diagnosed clinical stage II or III HR+/HER2+ breast cancer patients by the use of Palbociclib in combination with 
neoadjuvant letrozole and weekly trastuzumab.

Androgen receptor (AR) expression is significantly associated with $\mathrm{Rb}$ expression, which is disturbed by CDK4/6 inhibitors. Besides, $\mathrm{Rb}$ expression in TNBC was verified to improve tumor sensitivity to Erlotinib and Palbociclib Combination Therapy [31]. So as to confirm the role of CKIs in $\mathrm{AR}+/ \mathrm{TNBC}$ breast cancer, stage I-II trials NCT02605486 and BRE15-024 (NCT03090165) are surveying whether Palbociclib in combination with Bicalutamide improve the PFS, CBR of metastatic patients and whether Ribociclib with Bicalutamide promote CBR of TNBC, separately.

In the mentioned phase 2 study, four $(11 \%)$ of the 37 patients enrolled were TNBC, and all of them rapidly progressed under the Palbociclib treatment [3].

A case report showed that a high-grade primary neuroendocrine breast cancer patient underwent an ideal and durable response to Palbociclib in conjunction with endocrine therapy after non-effectiveness of commonly used platinum-based chemotherapy and endocrine therapy [32].

Although CDK4/6 inhibitors have a great tendency to bring about positive prognosis of HR+/HER2- breast cancer patients, for other type such as HER2+, TNBC or AR+ breast cancer, the application of CDK4/6 inhibitors still needs further intensive studies.

\section{Adverse effects of CDK4/6 inhibitors}

The summary of eight clinical trials illustrates that the first-line or later CDK4/6 inhibitor-based combination therapies bring about the definitely improved progression free survival (PFS), although with inevitable side effects. The most common side effects of the three CDK4/6 inhibitors are neutropenia, leukopenia, fatigue and nausea. Meanwhile, the most common grade 3/4 side effects are neutropenia, leukopenia and diarrhea. Interestingly, the neutropenia initiated by CDK4/6 inhibitors is rather different from chemotherapyinduced neutropenia for its rapid reversibility [33]. In detail, Abemaciclib has a 50\% lower neutropenia rate in contrast with Palbociclib and Ribociclib according to its better selectivity to CDK4. Therefore, patients receiving Palbociclib will mostly suffer from neutropenia, the Ribociclib from neutropenia, QTc prolongation and hepatobilary toxicity, and the Abemaciclib from gastrointestinal toxicity. Discomforts induced by the CKIs will be recovered rapidly by its reductions or interruptions [34].

Palbociclib was approved to apply in the treatment of advanced breast cancer in February 2015 in the USA. One year Palbociclib-use information post
US approval was collected to demonstrate the efficacy of Palbociclib in the real world. Of those, 612 advanced breast cancer patients received Palbociclib concomitantly with Letrozole. Through the complete blood count $(\mathrm{CBC})$ monitoring test during the first cycle of Palbociclib treatment, results came that $74.6 \%$ of patients experienced the neutropenia including $47.3 \%$ and $8.0 \%$ of them with a grade 3 or 4 occurrence, in consistence with the clinical trials PALOMA-2 (56\% and 10\%) and PALOMA-3 (55\% and $11 \%)$, respectively[35].

Moreover, ongoing phase II-IV clinical trials such as PALINA, NCT02600923, NCT02679755 and NCT03633331 are exploring the most frequent adverse effects resulting from the application of Palbociclib in HR+/HER2- advanced breast cancer patients. An ongoing non-randomized, phase I (NCT03065387) study will measure the safety of the pan-HER inhibitor neratinib in combination with Palbociclib in HER2 mutation/amplification advanced breast cancer patients.

The MONALEESA-2 trial also detected the most common adverse effects were neutropenia, leukopenia, and anemia, while elderly patients receiving Ribociclib showed a greater than $10 \%$ increase in the incidence of fatigue [11]. The phase II clinical trial LEADER and the phase I trial NCT02599363 are under research to deliver more side effects information of Ribociclib treatment in ER+ early breast cancer.

A case reported a 74-year-old breast cancer woman developed the radiation-induced morphea (RIM) after experiencing neoadjuvant CDK4/6 inhibitor Abemaciclib and aromatase inhibitor Anastrozole treatment in combination of the subsequent radiation therapy [36].

In contrast, the toxicity of Abemaciclib was much milder. At the same time, RB S780 phosphorylation inhibition and topoisomerase IIa abundance in skin biopsies as well as in tumor biopsies were newly detected biomarkers to reflect drug responses. Furthermore, reduction of RB phosphorylation by above $60 \%$ was predicted for the disease control [17]. In the Phase I trial of advanced ER+/HER2- breast cancer, the evaluation of Abemaciclib was performed along with a $72 \%$ disease control rate.

Two phase I clinical trials named NCT02057133 and NCT02779751 are studying the adverse effects in the combination of Abemaciclib with endocrine therapy.

\section{Interactions and combinations with other therapies}

All three CDK4/ 6 inhibitors can be orally taken and their metabolism will be influenced by CYP3A 
and SULT2A1 enzymes. Therefore, interactions with CYP3A inhibitors or CYP3A inducers may result in an increased toxicity or decreased efficacy of the three CDK4/ 6 inhibitors, respectively.

Palbociclib was confirmed to be well tolerated when combined with the c-MET inhibitor, Crizotinib, without causing significantly abnormal serum chemistry and hematology levels in vitro [37].

It is known that PFKFB3 inhibits ubiquitin proteasome degradation of the heat shock protein90Cdc37-CDK4 complex to increase CDK4 protein. Researchers found that disrupting the interaction of PFKFB3 and CDK4 by mutating lysine (147) to alanine led to accelerated degradations of CDK4. Therefore, the effect of Palbociclib will be improved by interfering PFKFB3-CDK4 interactions, probably by specific mutant approaches [38].

Triplet joint therapy of Ribociclib with exemestane and Everolimus (mTOR inhibitor), or Letrozole and Alpelisib (PI3Ka-selective inhibitor) were investigated in $\mathrm{HR}+\mathrm{ABC}$ patients whose tumor cells endured PI3K/AKT/mTOR and/or cyclin DCDK4/6-p16-Rb pathway alterations[39-41]. Pharmacokinetic indices for Ribociclib and Alpelisib were almost the same with those in their single-agent application. Moreover, after combined use of Ribociclib, the level of Everolimus, metabolized by cytochrome P450 3A4 (CYP3A4), increased by 1.5-to 3 -fold, which provided evidence to the possibility of lower doses of Everolimus in combination with Ribociclib, leading to less drug toxicity[42].

Okada et al. provided a novel antitumor strategy of combining CDK4 inhibitor with autophagy inhibition by either chloroquine (CQ) or knockdown of ATG5 or BECN1 to induce synthetic lethal toxicity for BT474, MDA-MB435S, SKBr3 breast cancer cells [43].

The impact of Abemaciclib on tumor immune microenvironment was investigated by in vitro and in vivo assays. Abemaciclib was reported to activate $\mathrm{T}$ cell inflammatory functions and antigen presentation genes in tumors. So a combination of Abemaciclib and anti-PD-L1 therapy, that modulate $\mathrm{T}$ cell anti-tumor immunity, was proven to delay of tumor growth [44].

The combination of Palbociclib, Ribociclib or Abemaciclib with other pathway molecule drugs is helpful to boost the therapy responses with decreased drug doses and tolerable toxicities.

\section{Resistance and solutions}

After continual application of CDK4/6 inhibitors, the unwanted acquired resistance is emerging. Therefore, we need to find potential post-CDK $4 / 6$ inhibitor therapeutic strategies to cope with its resistance [45].
Studies have reported that ESR1 mutation with ongoing trial's confirmation (NCT02738866) and loss of ER, upregulation of fibroblast growth factor receptor (FGFR) amplification, and acquired RB1 mutations [46] were in correlation with acquired CDK4/ 6 inhibitors resistance.

A clinical report compared the circulating tumor DNA (ctDNA) pre-exposure and after-exposure to CDK4/6 inhibitor (Palbociclib or Ribociclib) in three metastatic BC patients, discovering the substitution in donor splicing site of exon 8 of the RB1 gene, substitution in donor splicing site of exon 22 together with exon 19 deletion and exon 3 insertion, mutation in exon $16 \mathrm{H} 483 \mathrm{Y}$, respectively [46].

Combination of phosphatidylinositol-3 kinase (PI3K) or mammalian target of rapamycin (mTOR) inhibitors with CDK4/6 inhibitors and endocrine inhibitors has a tendency to prolong CDK4/6 inhibitor sensitivity [47].

\section{Palbociclib}

By using an ATP/ADP probe-based chemoproteomics platform, Nomanbhoy et al were able to predict whether a patient was resistant to palbociclib or not [48].

The efficacy of Palbociclib is limited by increased CDK2 and RBL1 expression [49]. In details, CCNE1 amplification upregulated levels of CDK2/CCNE1, following by RB inactivation and CDK4/ 6 inhibitors resistance [50]. Studies described synergy between CDK4 and PI3K inhibitors, for PI3K inhibitors would suppress cyclin D1 expression, leading to impediments to the presence of Palbociclib-resistant clones [51]. A preclinical study demonstrated that mechanisms of resistance to CDK4/6 inhibitors differ between Palbociclib and Ribociclib. In this study, Palbociclib-resistant cells presented increased cycle E protein levels while Ribociclib-resistant clones produced increased E2F1[52]. The consistent discovery was uncovered that resistance to Palbociclib was associated with non-luminal subtypes as well as persistent E2F-target gene expression [16].

The presence of T172-phosphorylated CDK4 was proved to predict the sensitivity to Palbociclib in breast cancer, which could help to select a subset of drug sensitive patients in case of inefficacy [53].

The ongoing phase IV NCT03401359 trial plans to recruit 100 metastatic breast cancer patients to discover biomarkers of acquired resistance response to Palbociclib from whole exome sequencing, RNASeq, circulating tumor DNA and flow cytometry approaches, thus making the targeted treatments more effective and flexible. 


\section{Ribociclib}

The transport of Ribociclib is affected by ABCB1 across the membranes and it has potential to deliver drug-drug interactions (DDIs) via ABCB1 and ABCG2 transporters as well as CYP isoforms, probably leading to beneficial MDR-reversing [54].

Risi and colleagues calculated from ten studies to detected a potentially predictive biomarker namely RBsig, a gene signature of RB loss. They found that low expression of RBsig separated a subset of ER+/HER2+ breast cancer patients with low pCR rates as they were resistant to neoadjuvant chemotherapy, anti-HER2 therapy and CDK4/6 inhibitor combinations [55].

Meanwhile, researchers were challenged to discover approaches to overcome the resistance to CDK4/6 inhibitors. PACE trial (NCT03147287) and MAINTAIN trial (NCT02632045) are ongoing to evaluate the efficacy of Ribociclib in HR+ MBC patients who have received CDK4/6 inhibitor before. TRINITI-1 trial (NCT01857193) explores the effects of triplet combination of Everolimus with Ribociclib and Exemestane-progressed patients who had prior use of CDK4/ 6 inhibitor. Also, NCT03238196 studies triplet combination of Erdafitinib with Ribociclib and Fulvestrant for patients with HR+ MBC [56].

The PI3K/AKT/mTOR pathway is well known as a critical pathway in HR positive breast cancer. So combination of Ribociclib with the PI3K inhibitor alpelisib (BYL719) brought about enhanced tumor regression, response rates and PFS versus single-agent treatment [57-59]. But recent data indicated that the application of PI3K inhibitors seems disappointing because of its modest effects and great toxicities, while the mTOR inhibitor everolimus evidently improves PFS when added to ET with less toxicity [60]. Also, recent data shows that CDK4/6 inhibitor-resistant breast cancer cell lines tend to reactivate the CDK-RB-E2F pathway, but are sensitive to the mTORC1/2 inhibitors [61]. Therefore, combination of an mTOR or mTORC1/2 inhibitor with a CDK4/6 inhibitor will produce more effects on E2F-dependent transcription as well as regressions of cell growths, which overcome the resistance to the sole use of CDK4/6 inhibitors and delay initiation of chemotherapy.

\section{Abemaciclib \\ TP53 mutations may be a potential biomarker to predict an Abemaciclib-nonresponding type of breast cancer patients [17]. \\ In brief, more effective biomarkers for CDK4/ 6 inhibitors resistant predictors are needed to select the best available treatment and generate better prognosis in the rather poor type of breast cancer patients.}

\section{Discussion}

In the case of HR+/HER2- breast cancer, endocrine therapy is usually the first-line therapy depending on the menopausal status of the patient. However, acquired resistance to endocrine treatments is in desperate need of effective solutions. Therefore several alternate choices including high-dose Fulvestrant or Everolimus, PI3K inhibitors, Entinostat or CDK 4/6 inhibitors are under research $[62,63]$.

Multiple evidences have held the critical role of CDK-RB-E2F pathway in cell proliferation of breast cancer [64]. Preclinical evidence demonstrated that the interruption of cyclin $\mathrm{D} 1 / \mathrm{CDK} 4 / 6 / \mathrm{Rb}$ axis in breast cancer encountered the disruption of cell cycle. The addition of CDK4/6 inhibitors to endocrine therapy have produced increased efficacy and improved PFS. And three CDK4/6 inhibitors (Palbociclib, Ribociclib and Abemaciclib) have been approved by the US Food and Drug Administration [65-68]. The three CDK inhibitors bind to CDKs family and interrupt the binding of ATP. They form hydrogen bonds with CDKs and interact with CS6 and CS7 on the catalytic spine, playing a significant role in the regulation of cell cycle [69].

Palbociclib (PD0332991) is the first oral CDK4/6 inhibitor with a simultaneous use of letrozole as first-line therapy, resulting in the significantly improved median PFS in advanced breast cancer with ER positive and HER2 negative (24.8 vs 14.5 months [PALOMA2]) as well as with Fulvestrant in endocrine pretreated patients (9.2 vs 3.8 months [PALOMA-3]) $[70,71]$.

MONARCH 1, 2, and 3 clinical trials established Abemaciclib as an orally effective CDK4/6 inhibitor for HR+/HER2- advanced or metastatic breast cancer $[72,73]$.

Up to now, researchers have adopted sequential administration of the pan-CDK inhibitor Roscovitine following by doxorubicin to TNBC cells, resulting in increased DNA damages, reduced recruitment of homologous recombination proteins with decreased tumor size, improved overall survival in comparison with single or concomitant treatment in TNBC [74]. Therefore, the application of CDK4/ 6 inhibitors might also produce similar prognosis in TNBC and other subtype of breast cancer while several ongoing studies are under implementation.

With splendid advantages of the addition use of CDK4/ 6 inhibitors, adverse effects are unwelcomed but inevitable. For example, Palbociclib mainly causes bone marrow suppression however it is always reversible by absence or withdrawal of Palbociclib, clearly differentiated from the apoptotic cell death caused by chemotherapy [75]. 
Recently, radiotherapy plays a more and more vital role in the comprehensive treatment of breast cancer patients [76]. Besides, latest literature has discussed the effect of Ribociclib in combination with radiotherapy in head and neck squamous cell carcinoma. Results suggested that Ribociclib was a potential radiosensitizer by enhancing the cytotoxic effects of radiation in OML1 cells and reversing the radiation resistance in OML1-resistent cells. So evidences on Ribociclib during radiotherapy for breast cancer cells should be collected and taken into consideration [77].

Interestingly, CDK4/6 inhibitors might display influences on the tumor microenvironment. For instance, inhibition of CDK4 activity would significantly reduce the stabilization of autophagy-mediated proteasome NOXA and induce cell apoptosis in mantle cell lymphoma [78]. Also, studies demonstrated that cyclin D-CDK4 kinase destabilized PD-L1 via Cullin3 ${ }^{\text {SPOP }}$ to control cancer immune surveillance and revealed the potential combination of CDK4/6 inhibitors and PD-1/PD-L1 blockade to enhance therapeutic efficacy for human cancers [79]. Simultaneously, combined targeting of MDM2 and CDK4 was synergistic in dedifferentiated liposarcomas. The use of RG7388 and Palbociclib together was found to exert a greater anti-tumor effect than either drugs alone [80]. New findings will be helpful to emphasize the prevalent action of CDK4/6 inhibitors throughout the treatment process in breast cancer patients.

Overall we skimmed researches concerning the efficacy and safety of Palbociclib, Ribociclib and Abemaciclib in diverse types of breast cancer patients. Although CDK4/6 inhibitors generate side effects especially hematologic changes, their distinct superiority is more glaring in breast cancer treatments. So with inevitable resistant mechanisms, more and more clinical trials are seeking for potential biomarkers to predict the validity of the CDK4/6 inhibitors, thus bringing in more optionally effective therapy strategies to breast cancer patients, even to the currently incurable breast cancer patients.

\section{Acknowledgements}

\section{Funding statement}

This research was supported by the National Key Research and Development Program of China (No. 2016YFC0905900), National Natural Science Foundation of China (No. 81902987), and Postgraduate Research and Practice Innovation Program of Jiangsu Province (KYCX18_1490 and KYCX18_1485).

\section{Competing Interests}

The authors have declared that no competing interest exists.

\section{References}

1. Steger G G, Gnant M, Bartsch R. Palbociclib for the treatment of postmenopausal breast cancer - an update[J]. Expert Opinion On Pharmacotherapy. 2016; 17(2):255-63.

2. Rugo HS, Vidula N, Ma C. Improving Response to Hormone Therapy in Breast Cancer: New Targets, New Therapeutic Options[J]. American Society of Clinical Oncology educational book. 2016; 35: e40-54. doi: 10.14694/EDBK_159198.

3. DeMichele A, Clark AS, Tan KS, et al. CDK 4/6 inhibitor palbociclib (PD0332991) in Rb+ advanced breast cancer: phase II activity, safety, and predictive biomarker assessment[J]. Clinical Cancer Research. 2015; 21(5):995-1001.

4. Sarosiek T. Inhibitors of cyclin-dependent kinases (CDK) - a new group of medicines in therapy of advanced breast cancer[J]. Polski Merkuriusz Lekarski. 2018; 44(259):5-9.

5. Iwata H. Clinical development of CDK4/6 inhibitor for breast cancer[J]. Breast Cancer. 2018; 25(4):402-406

6. $\mathrm{Xu} \mathrm{H}, \mathrm{Yu} \mathrm{S}$, Liu $\mathrm{Q}$, et al. Recent advances of highly selective CDK4/6 inhibitors in breast cancer[J]. Journal Of Hematology \& Oncology. 2017; 24;10(1):97.

7. Whittaker S R, Mallinger A, Workman P, et al. Inhibitors of cyclin-dependent kinases as cancer therapeutics[J]. Pharmacology \& therapeutics. 2017; 173: 83-105.

8. [No authors listed]. Ribociclib Approved for Advanced Breast Cancer[J]. Cancer Discovery. 2017; 7(5):OF3. doi: 10.1158/2159-8290.

9. Hecht K A, Selby C. Review of Cyclin-Dependent Kinase 4/6 Inhibitors for the Treatment of Hormone Receptor-Positive Advanced Breast Cancer[J]. The Annals Of Pharmacotherapy. 2019; 53(2):195-203.

10. Syed Y Y. Ribociclib: First Global Approval[J]. Drugs. 2017; 77(7):799-807.

11. Sonke G S, Hart L L, Campone M, et al. Ribociclib with letrozole vs letrozole alone in elderly patients with hormone receptor-positive, HER2-negative breast cancer in the randomized MONALEESA-2 trial[J]. Breast Cancer Research and Treatment. 2018; 167(3):659-669.

12. O'Shaughnessy J, Petrakova K, Sonke G S, et al. Ribociclib plus letrozole versus letrozole alone in patients with de novo HR+, HER2- advanced breast cancer in the randomized MONALEESA-2 trial[J]. Breast Cancer Research and Treatment. 2018; 168(1):127-134

13. Mistry R, Suri G, Young K, et al. Budget impact of including ribociclib in combination with letrozole on US payer formulary: first-line treatment of post-menopausal women with HR+/HER2- advanced or metastatic breast cancer[J]. Current Medical Research And Opinion. 2018; 34(12):2143-2150.

14. Mistry R, May J R, Suri G, et al Cost-Effectiveness of Ribociclib plus Letrozole Versus Palbociclib plus Letrozole and Letrozole Monotherapy in the First-Line Treatment of Postmenopausal Women with HR+/HER2- Advanced or Metastatic Breast Cancer: A U.S. Payer Perspective[J]. Journal Of Managed Care \& Specialty Pharmacy. 2018; 24(6):514-523.

15. Curigliano G, Gómez Pardo P, Meric-Bernstam F, et al. Ribociclib plus letrozole in early breast cancer: A presurgical, window-of-opportunity study[J]. Breast. 2016;28:191-8.

16. Ma C X, Gao F, Luo J, et al. NeoPalAna: Neoadjuvant Palbociclib, a Cyclin-Dependent Kinase 4/6 Inhibitor, and Anastrozole for Clinical Stage 2 or 3 Estrogen Receptor-Positive Breast Cancer[J]. Clinical Cancer Research. 2017; 23(15):4055-4065.

17. Patnaik A, Rosen L S, Tolaney S M, et al. Efficacy and Safety of Abemaciclib, an Inhibitor of CDK4 and CDK6, for Patients with Breast Cancer, Non-Small Cell Lung Cancer, and Other Solid Tumors[J]. Cancer Discovery. 2016; 6(7):740-53.

18. Barroso-Sousa R, Shapiro G I, Tolaney S M. Clinical Development of the CDK4/6 Inhibitors Ribociclib and Abemaciclib in Breast Cancer[J]. Breast Care (Basel). 2016:11(3):167-73

19. Fujiwara Y, Tamura K, Kondo S, et al. Phase 1 study of abemaciclib, an inhibitor of CDK 4 and 6, as a single agent for Japanese patients with advanced cancer[J]. Cancer Chemotherapy And Pharmacology. 2016; 78(2):281-8.

20. Kwapisz D. Cyclin-dependent kinase 4/6 inhibitors in breast cancer: palbociclib, ribociclib, and abemaciclib[J]. Breast Cancer Research And Treatment. 2017; 166(1):41-54

21. Bashour SI, Doostan I, Keyomarsi K, et al. Rapid Breast Cancer Disease Progression Following Cyclin Dependent Kinase 4 and 6 Inhibitor Discontinuation[J]. Journal of Cancer. 2017; 8(11):2004-2009.

22. Owsley J, Jimeno A, Diamond J R. Palbociclib:CDK4/6 inhibition in the treatment of ER-positive breast cancer[J]. Drugs Of Today (Barc). 2016; 52(2):119-29.

23. Princic N, Aizer A, Tang D H, et al. Predictors of systemic therapy sequences following a CDK $4 / 6$ inhibitor-based regimen in post-menopausal women with hormone receptor positive, HEGFR- 2 negative metastatic breast cancer[J]. Current Medical Research And Opinion. 2018: 1-8. doi: 10.1080/03007995.2018.1519500. 
24. Stearns V, Brufsky A M, Verma S, et al. Expanded-Access Study of Palbociclib in Combination With Letrozole for Treatment of Postmenopausal Women With Hormone Receptor-Positive, HER2-Negative Advanced Breast Cancer[J]. Clinical Breast Cancer. 2018; 18(6):e1239-e1245.

25. Dhakal A, Matthews C M, Levine E G, et al. Efficacy of Palbociclib Combinations in Hormone Receptor-Positive Metastatic Breast Cancer Patients After Prior Everolimus Treatment[J]. Clinical Breast Cancer. 2018. 18(6):e1401-e1405

26. Abraham J, Coleman R, Elias A, et al. Use of cyclin-dependent kinase (CDK) $4 / 6$ inhibitors for hormone receptor-positive, human epidermal growth factor receptor 2-negative, metastatic breast cancer: a roundtable discussion by The Breast Cancer Therapy Expert Group (BCTEG) [J]. Breast Cancer Research And Treatment. 2018; 171(1):11-20.

27. Curigliano G, Criscitiello C, Esposito A, et al. Pharmacokinetic drug evaluation of ribociclib for the treatment of metastatic, hormone-positive breast cancer[J]. Expert Opinion On Drug Metabolism \& Toxicology. 2017; 13(5):575-581.

28. Witkiewicz A K, Cox D, Knudsen E S. CDK4/6 inhibition provides a potent adjunct to Her2-targeted therapies in preclinical breast cancer models[J]. Genes Cancer. 2014; 5(7-8):261-72

29. Asghar U S, Barr A R, Cutts R, et al. Single-Cell Dynamics Determines Response to CDK4/6 Inhibition in Triple-Negative Breast Cancer[J]. Clinical Cancer Research. 2017; 23(18):5561-5572.

30. Matutino A, Amaro C, Verma S. CDK4/6 inhibitors in breast cancer: beyond hormone receptor-positive HER2-negative disease[J]. Therapeutic Advances In Medical Oncology. 2018; 10:1758835918818346. doi: $10.1177 / 1758835918818346$.

31. Gianni L, Bisagni G, Colleoni $\mathrm{M}$, et al. Neoadjuvant treatment with trastuzumab and pertuzumab plus palbociclib and fulvestrant in HER2-positive, ER-positive breast cancer (NA-PHER2): an exploratory, open-label, phase 2 study[J]. Lancet Oncology. 2018; 19(2):249-256.

32. Foidart P, Yip C, Radermacher J, et al. Expression of MT4-MMP, EGFR, and RB in Triple-Negative Breast Cancer Strongly Sensitizes Tumors to Erlotinib and Palbociclib Combination Therapy[J]. Clinical Cancer Research. 2019; 25(6):1838-1850.

33. Shanks A, Choi J, Karur V. Dramatic response to cyclin D-dependent kinase $4 / 6$ inhibitor in refractory poorly differentiated neuroendocrine carcinoma of the breast[J]. Proceedings (Baylor University Medical Center). 2018; 31(3):352-354.

34. Spring L M, Zangardi M L, Moy B, et al. Clinical Management of Potential Toxicities and Drug Interactions Related to Cyclin-Dependent Kinase 4/6 Inhibitors in Breast Cancer: Practical Considerations and Recommendations[J]. Oncologist. 2017; 22(9):1039-1048.

35. Thill M, Schmidt M. Management of adverse events during cyclin-dependent kinase 4/6 (CDK4/6) inhibitor-based treatment in breast cancer[J]. Therapeutic Advances In Medical Oncology. 2018; 10:1758835918793326. doi: $10.1177 / 1758835918793326$

36. Kish J K, Ward M A, Garofalo D, et al. Real-world evidence analysis of palbociclib prescribing patterns for patients with advanced/metastatic breast cancer treated in community oncology practice in the USA one year post approval[J]. Breast Cancer Research. 2018; 20(1):37.

37. Partl R, Regitnig P, Tauber G, et al. Radiation-induced morphea-a rare but severe late effect of adjuvant breast irradiation: Case report and review of the literature[J]. Strahlentherapie Und Onkologie. 2018; 194(11):1060-1065.

38. Smith B, Hsu Y H, Flores R, et al. Single oral dose acute and subacute toxicity of a c-MET tyrosine kinase inhibitor and CDK $4 / 6$ inhibitor combination drug therapy[J]. American Journal Of Cancer Research. 2018; 8(1):183-191.

39. Jia $W$, Zhao $X$, Zhao L, et al. Non-canonical roles of PFKFB3 in regulation of cell cycle through binding to CDK4[J]. Oncogene. 2018; 37(13):1685-1698.

40. [Internet] Juric D, Ismail-Khan R, Campone M, et al. Abstract P8-12: Phase $\mathrm{Ib} / \mathrm{II}$ study of ribociclib and alpelisib and letrozole in ER+, HER2- advanced breast cancer: Safety, preliminary efficacy, and molecular analysis. The Thirty-Eighth San Antonio Breast Cancer Symposium. 2015. https://www.sabcs.org.

41. Bardia A, Modi S, Oliveira M, et al. Abstract P6-13-01: Triplet therapy with ribociclib, everolimus, and exemestane in women with HR+/HER2-advanced breast cancer. San Antonio Breast Cancer Symposium; 2016. DOI: 10.1158/1538-7445.

42. Bhansali S, Parasuraman S, Hewes B,et al. P.14-6: Application of SimCyp® and real-time pharmacokinetic assessments in defining dose in novel-novel combinations with potential drug-drug interactions in cancer patients. Proceedings of the 2014 Annual Meeting American College of Clinical Pharmacology; 2014. https://accp1.org/documents/ACCP2014program-min.

43. Okada Y, Kato S, Sakamoto Y, et al. Synthetic lethal interaction of CDK inhibition and autophagy inhibition in human solid cancer cell lines[J]. Oncology Reports. 2017; 38(1):31-42.

44. Schaer D A, Beckmann R P, Dempsey J A, et al. The CDK4/6 Inhibitor Abemaciclib Induces a $\mathrm{T}$ Cell Inflamed Tumor Microenvironment and Enhances the Efficacy of PD-L1 Checkpoint Blockade[J]. Cell Reports. 2018; 22(11):2978-2994.

45. O'Leary B, Finn R S, Turner N C. Treating cancer with selective CDK4/6 inhibitors[J]. Nature reviews Clinical oncology. 2016; 13(7):417-30.

46. Condorelli R, Spring L, O'Shaughnessy J, et al. Polyclonal RB1 mutations and acquired resistance to $\mathrm{CDK} 4 / 6$ inhibitors in patients with metastatic breast cancer[J]. Annals of Oncology. 2018; 29(3):640-645.
47. Cortes I, Im S A, Holgado E, et al. The next era of treatment for hormone receptor-positive, HER2-negative advanced breast cancer: Triplet combination-based endocrine therapies[J]. Cancer Treatment Reviews. 2017; 61: 53-60.

48. Nomanbhoy T K, Sharma G, Brown H, et al. Chemoproteomic Evaluation of Target Engagement by the Cyclin-Dependent Kinase 4 and 6 Inhibitor Palbociclib Correlates with Cancer Cell Response[J]. Biochemistry. 2016; 55(38):5434-41.

49. Dean J L, Thangavel C, McClendon A K, et al. Therapeutic CDK4/6 inhibition in breast cancer: key mechanisms of response and failure[J]. Oncogene. 2010;29(28):4018-32.

50. Herrera-Abreu M T, Palafox M, Asghar U, et al. Early Adaptation and Acquired Resistance to CDK4/6 Inhibition in Estrogen Receptor-Positive Breast Cancer[J]. Cancer Research. 2016;76(8):2301-13.

51. Vora S R, Juric D, Kim N, et al. CDK 4/6 inhibitors sensitize PIK3CA mutant breast cancer to PI3K inhibitors[J]. Cancer Cell. 2014;26(1):136-49.

52. Lenihan $C$, Bouchekioua-Bouzaghou $K$, Abdulghani $R$, et al. Abstract P3-03-12: CDK4/6 inhibitor resistant ER-positive cells remain dependent on estrogen signalling and retain sensitivity to endocrine therapy. San Antonio Breast Cancer Symposium; 2016. DOI: 10.1158/1538-7445.

53. Raspe E, Coulonval K, Pita J M, et al. CDK4 phosphorylation status and a linked gene expression profile predict sensitivity to palbociclib[J]. EMBO Molecular Medicine. 2017; 9(8):1052-1066.

54. Sorf A, Hofman J, Kucera R, et al. Ribociclib shows potential for pharmacokinetic drug-drug interactions being a substrate of $\mathrm{ABCB} 1$ and potent inhibitor of ABCB1, ABCG2 and CYP450 isoforms in vitro[J]. Biochemical Pharmacology. 2018; 154:10-17.

55. Risi E, Grilli A, Migliaccio I, et al. A gene expression signature of Retinoblastoma loss-of-function predicts resistance to neoadjuvant chemotherapy in ER-positive/HER2-positive breast cancer patients[J]. Breast Cancer Research And Treatment. 2018; 170(2):329-341.

56. Spring L, Bardia A. Cycling Toward Progress: Ribociclib, a CDK 4/6 Inhibitor for Breast Cancer[J]. Clinical Cancer Research. 2018; 24(13):2981-2983.

57. Parasuraman S, Caponigro G, Loo A, et al. P. 5-7: LEE011, a potent and selective CDK4/6 inhibitor, under preclinical and clinical investigation. Proceedings of the 12th International Congress on Targeted Anticancer Therapies; 2014.

58. Gao $\mathrm{H}$, Korn J M, Ferretti $\mathrm{S}$, et al. High-throughput screening using patient-derived tumor xenografts to predict clinical trial drug response[J]. Nature Medicine. 2015; 21(11):1318-25.

59. O'Brien N A, Tomaso E D, Ayala R, et al. Abstract 4756: In vivo efficacy of combined targeting of CDK4/6, ER and PI3K signaling in ER+ breast cancer. Proceedings: AACR Annual Meeting; 2014. DOI: 10.1158/1538-7445.

60. Ballinger T J, Meier J B, Jansen V M. Current Landscape of Targeted Therapies for Hormone-Receptor Positive, HER2 Negative Metastatic Breast Cancer[J]. Frontiers In Oncology. 2018; 8: 308.

61. Michaloglou C, Crafter C, Siersbaek R, et al. Combined Inhibition of mTOR and CDK4/6 Is Required for Optimal Blockade of E2F Function and Long-term Growth Inhibition in Estrogen Receptor-positive Breast Cancer[J]. Molecular Cancer Therapeutics. 2018; 17(5):908-920.

62. Perez E A. Treatment strategies for advanced hormone receptor-positive and human epidermal growth factor 2-negative breast cancer: the role of treatment order[J]. Drug resistance updates. 2016; 24:13-22.

63. Hamilton E, Infante J R. Targeting CDK4/6 in patients with cancer[J]. Cancer Treatment Reviews. 2016; 45: 129-38.

64. Johnson J, Thijssen B, McDermott U, et al. Targeting the RB-E2F pathway in breast cancer[J]. Oncogene. 2016; 35(37):4829-35.

65. Scott S C, Lee S S, Abraham J. Mechanisms of therapeutic CDK4/6 inhibition in breast cancer[J]. Seminars In Oncology. 2017; 44(6):385-394.

66. Sammons S L, Topping D L, Blackwell K L. HR+, HER2- Advanced Breast Cancer and CDK4/6 Inhibitors: Mode of Action, Clinical Activity, and Safety Profiles[J]. Current Cancer Drug Targets. 2017; 17(7):637-649.

67. Echavarria I, Jerez Y, Martin M, et al. Incorporating CDK4/6 Inhibitors in the Treatment of Advanced Luminal Breast Cancer[J]. Breast Care (Basel). 2017; 12(5):296-302.

68. Polk A, Kolmos I L, Kumler I, et al. Specific CDK4/6 inhibition in breast cancer: a systematic review of current clinical evidence[J]. ESMO open. 2016; 1(6):e000093.

69. Roskoski R Jr. Cyclin-dependent protein kinase inhibitors including palbociclib as anticancer drugs[J]. Pharmacological Research. 2016; 107:249-275

70. Schmidt M, Sebastian M. Palbociclib-The First of a New Class of Cell Cycle Inhibitors[J]. Recent Results In Cancer Research. 2018; 211: 153-175.

71. Chen F, Zhang J, Xu W, et al. Progress of CDK4/6 Inhibitor Palbociclib in the Treatment of Cancer[J]. Anticancer Agents In Medicinal Chemistry. 2018;18(9):1241-1251.

72. Palumbo A, Lau G, Saraceni M. Abemaciclib: The Newest CDK4/6 Inhibitor for the Treatment of Breast Cancer[J]. The Annals of Pharmacotherapy. 2019;53(2):178-185.

73. Corona S P, Generali D. Abemaciclib: a CDK4/6 inhibitor for the treatment of HR+/HER2- advanced breast cancer[J]. Drug Design, Development And Therapy. 2018; 12: 321-330.

74. Jabbour-Leung N A, Chen X, Bui T, et al. Sequential Combination Therapy of CDK Inhibition and Doxorubicin Is Synthetically Lethal in p53-Mutant 
Triple-Negative Breast Cancer[J]. Molecular Cancer Therapeutics. 2016; 15(4):593-607.

75. Hu W, Sung T, Jessen B A, et al. Mechanistic Investigation of Bone Marrow Suppression Associated with Palbociclib and its Differentiation from Cytotoxic Chemotherapies[]]. Clinical Cancer Research. 2016; 22(8):2000-8.

76. Castaneda S A, Strasser J. Updates in the Treatment of Breast Cancer with Radiotherapy[J]. Surgical Oncology Clinics Of North America. 2017; 26(3):371-382.

77. Tai T S, Lin P M, Wu C F, et al. CDK4/6 Inhibitor LEE011 Is a Potential Radiation-sensitizer in Head and Neck Squamous Cell Carcinoma: An In Vitro Study[J]. Anticancer Research. 2019; 39(2):713-720.

78. Heine S, Kleih M, Giménez N, et al. Cyclin D1-CDK4 activity drives sensitivity to bortezomib in mantle cell lymphoma by blocking autophagy-mediated proteolysis of NOXA[J]. Journal Of Hematology \& Oncology. 2018;11(1):112.

79. Zhang J, Bu X, Wang H, et al. Cyclin D-CDK4 kinase destabilizes PD-L1 via cullin 3-SPOP to control cancer immune surveillance[J]. Nature. 2018;553(7686):91-95.

80. Laroche-Clary A, Chaire V, Algeo M P, et al. Combined targeting of MDM2 and CDK4 is synergistic in dedifferentiated liposarcomas[J]. Journal Of Hematology \& Oncology. 2017; 10(1):123. 